\title{
Loss of PSD-95 Enrichment Is Not a Prerequisite for Spine Retraction
}

\author{
Georgia F. Woods, Won Chan Oh, Lauren C. Boudewyn, Sarah K. Mikula, and Karen Zito \\ Center for Neuroscience, University of California, Davis, California 95618
}

Changes in neuronal structure are thought to underlie long-term behavioral modifications associated with learning and memory. In particular, considerable evidence implicates the destabilization and retraction of dendritic spines along with the loss of spine synapses as an important cellular mechanism for refining brain circuits, yet the molecular mechanisms regulating spine elimination remain illdefined. The postsynaptic density protein, PSD-95, is highly enriched in dendritic spines and has been associated with spine stability. Because spines with low levels of PSD-95 are more dynamic, and the recruitment of PSD-95 to nascent spines has been associated with spine stabilization, we hypothesized that loss of PSD-95 enrichment would be a prerequisite for spine retraction. To test this hypothesis, we used dual-color time-lapse two-photon microscopy to monitor rat hippocampal pyramidal neurons cotransfected with PSD-95-GFP and DsRed-Express, and we analyzed the relationship between PSD-95-GFP enrichment and spine morphological changes. Consistent with our hypothesis, we found that the majority of spines that retracted were relatively unenriched for PSD-95-GFP. However, in the subset of PSD-95-GFP-enriched spines that retracted, spine shrinkage and loss of PSD-95-GFP were tightly coupled, suggesting that loss of PSD-95-GFP enrichment did not precede spine retraction. Moreover, we found that, in some instances, spine retraction resulted in a significant enrichment of PSD-95-GFP on the dendritic shaft. Our data support a model of spine retraction in which loss of PSD-95 enrichment is not required prior to the destabilization of spines.

\section{Introduction}

Novel sensory experience and mastery of new tasks have been associated with increased formation of dendritic spines, and also with increased spine loss, supporting the idea that these structural changes are important mechanisms that allow the brain to adapt to environmental stimuli (Xu et al., 2009; Yang et al., 2009; Roberts et al., 2010). Notably, extensive spine loss and synapse elimination occur during the development of a diverse set of brain regions (Wise et al., 1979; Rakic et al., 1986; Markus and Petit, 1987; De Felipe et al., 1997; Holtmaat et al., 2005; Zuo et al., 2005), and this refinement of circuitry coincides with increased behavioral competence (Lawrence and Hopkins, 1976; Rakic et al., 1986). These data suggest that elimination of spine synapses plays a critical role in mediating the circuit changes that underlie adaptive plasticity of the brain, yet the cellular and molecular mechanisms that lead to spine loss remain ill defined.

One molecule that has been linked to spine stability is the postsynaptic density protein, PSD-95. Studies using PSD-95 fused to green fluorescent protein (PSD-95-GFP) have primarily

\footnotetext{
Received Dec. 20, 2010; revised June 14, 2011; accepted July 5, 2011.

Author contributions: G.F.W., W.C.O., and K.Z. designed research; G.F.W., W.C.O., L.C.B., and S.K.M. performed research; G.F.W., W.C.O., and L.C.B. analyzed data; G.F.W. and K.Z. wrote the paper.

This work was supported by the Achievement Rewards for College Scientists Foundation (G.F.W.), The Hellman Family Foundation (K.Z.), the NIH (K.Z.), and a Burroughs Wellcome Fund Career Award in the Biomedical Sciences (K.Z.). We thank Julie Heiner, A. Cade Grunst, and Temesgen Woldeyesus for help with experiments and analysis, Tim $0^{\prime}$ Connor and Travis Hill for programming, and Drs. Elva Diaz, Kim McAllister, Johannes Hell, Mark Lucanic, and members of the Zito Laboratory for critical reading of this manuscript.

Correspondence should be addressed to Karen Zito, Center for Neuroscience, University of California, Davis, Davis, CA 95618.E-mail: kzito@ucdavis.edu.

DOI:10.1523/JNEUROSCI.6662-10.2011

Copyright $\odot 2011$ the authors $\quad 0270-6474 / 11 / 3112129-10 \$ 15.00 / 0$
}

focused on using PSD-95-GFP as a marker for assessing postsynaptic density (PSD) assembly as it relates to spine formation (Okabe et al., 2001; Prange and Murphy, 2001; De Roo et al., 2008). These studies support a model in which PSD-95 is recruited to new spines in an activity-dependent manner, where it contributes to the stabilization of nascent spines. Indeed, spines with low levels of PSD-95-GFP are more dynamic (Prange and Murphy, 2001; Ehrlich et al., 2007) and activity-dependent alterations in spine shape are regulated by PSD-95 (Steiner et al., 2008). Moreover, spines that are enriched for PSD-95-GFP are almost always juxtaposed to presynaptic markers, suggesting that these spines are incorporated into synapses (Prange and Murphy, 2001), and recent ultrastructural data support a key role for PSD-95 in maintaining the integrity of the PSD (Chen et al., 2011). As adaptive circuit changes must be associated with the retraction of spines that were once engaged in synapses, these data suggest that destabilization of dendritic spines via loss of PSD-95 enrichment might be a prerequisite for spine retraction.

To determine whether spine retraction occurs only after a significant loss of PSD-95 enrichment, we simultaneously monitored spine morphology and PSD-95-GFP levels over time using dual-color time-lapse two-photon imaging of hippocampal pyramidal neurons cotransfected with PSD-95-GFP and DsRedExpress. By quantifying changes in spine size and PSD-95-GFP levels during and after spine retraction, we found that, while the majority of spines that retracted exhibited low enrichment of PSD-95-GFP, a significant proportion had PSD-95-GFP enrichment levels comparable with that of persistent neighboring spines. In this subset of enriched spines, spine shrinkage and loss of PSD-95-GFP were tightly coupled, suggesting that loss of 
PSD-95 enrichment is not a prerequisite for spine retraction. In fact, in some cases spine retraction resulted in a significant enrichment of PSD-95-GFP on the dendritic shaft. Our data strongly support a model in which the retraction of dendritic spines is not dependent upon a significant loss of enrichment for PSD-95.

\section{Materials and Methods}

Preparation and transfection of organotypic slice cultures. Organotypic hippocampal slice cultures were prepared from P5 to P7 rats (both male and female) as described previously (Stoppini et al., 1991) in accordance with animal care and use guidelines of the University of California. Genes were delivered at 5-8 DIV using particle-mediated gene transfer, as described previously (Zito et al., 2004; Woods and Zito, 2008), except $4 \mu \mathrm{g}$ of PSD-95-GFP (Gray et al., 2006) and/or $10 \mu \mathrm{g}$ of DsRedExpress (Clontech) were coated onto $8 \mathrm{mg}$ of $1.6 \mu \mathrm{m}$ gold beads.

Two-photon time-lapse imaging. Pyramidal neurons were imaged $24-48 \mathrm{~h}$ after transfection using a custom two-photon microscope with a pulsed Ti::sapphire laser (Mai Tai; Spectra Physics) tuned to $930 \mathrm{~nm}$, a wavelength that simultaneously excites DsRed-Express and PSD-95-GFP. The microscope was controlled with ScanImage (Pologruto et al., 2003). For each neuron, image stacks $(512 \times 512$ pixels; $\sim 0.07 \mu \mathrm{m} /$ pixel) with $1 \mu \mathrm{m}$ steps were collected from four to eight segments of secondary dendrites (apical and basal). For time-lapse with $1 \mathrm{~h}$ intervals, slices were imaged at room temperature in culture medium and were returned to the incubator $\left(35^{\circ} \mathrm{C}\right)$ between imaging sessions. For time-lapse with intervals of seconds to minutes, slices were imaged at $30^{\circ} \mathrm{C}$ in artificial CSF (ACSF) containing the following (in $\mathrm{mm}$ ): $127 \mathrm{NaCl}, 25 \mathrm{NaHCO}_{3}, 25 \mathrm{D}$-glucose, $2.5 \mathrm{KCl}, 1.25 \mathrm{NaH}_{2} \mathrm{PO}_{4}, 1 \mathrm{MgCl}_{2}$, and $2 \mathrm{CaCl}_{2}$, aerated with $95 \% \mathrm{O}_{2} / 5 \% \mathrm{CO}_{2}$. Where indicated, $(R S)$-3-(2-carboxypiperazin-4-yl)-propyl-1-

phosphonic acid (CPP) (10 $\mu \mathrm{m}$ final; Tocris) was added directly to the ACSF. Red and green fluorescence photons were separated using a dichroic mirror $(565 \mathrm{~nm})$ and bandpass filters (BG22 glass and 607/45 nm; Chroma) and collected using photomultiplier tubes (R3896; Hamamatsu). Epifluorescence and transfluorescence signals were summed. All images shown are maximum projections of 3D stacks unless otherwise stated.

Quantification of spine densities and spine dynamics. All distinct protrusions emanating from the dendritic shaft, regardless of shape, were counted and measured in images from the red (DsRed-Express) channel using custom software written in MATLAB. As widespread spine loss can occur when cell health is compromised, we monitored spine density during time-lapse imaging. For all cells included in our analysis, overall spine density did not significantly decrease over the $2 \mathrm{~h}$ imaging session (data not shown). This ensured that the spontaneous spine retractions being examined did not take place due to a general decrease in cell health.

Quantification of fluorescence intensities in spines and dendrites. Integrated red and green fluorescence intensities were measured from boxed regions of interest (ROIs) $\left(\sim 0.5 \mu \mathrm{m}^{2}\right)$ on the dendrites and from boxed ROIs $\left(\sim 1.0 \mu \mathrm{m}^{2}\right)$ surrounding the spine heads in the single $Z$ stack image slice in which the spine appeared brightest. All spine and dendrite fluorescence intensities were bleed-through corrected and background subtracted. Bleed-through of green into the red channel and red into the green channel, as determined by quantifying red and green signal inten-
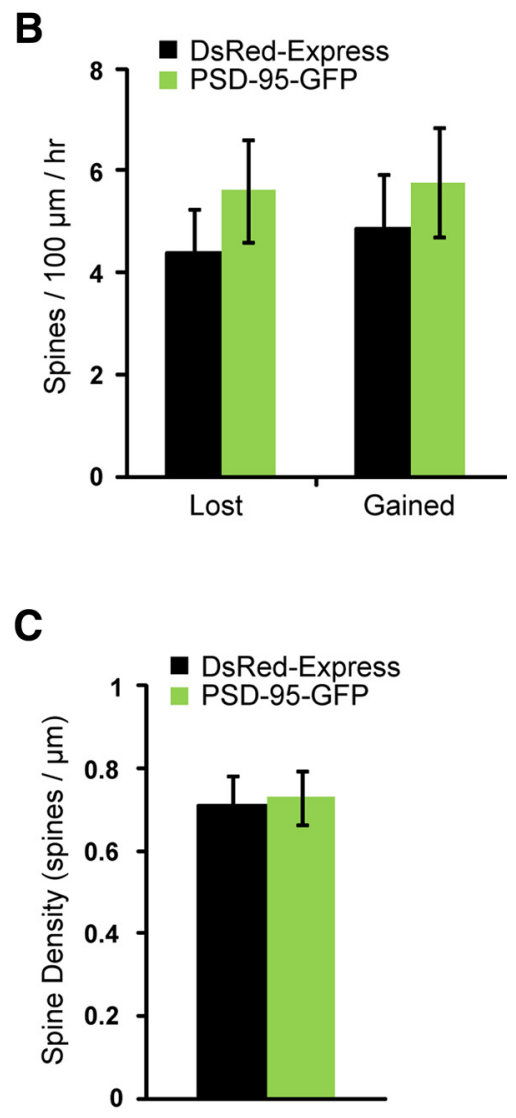

$60 \mathrm{~min}$
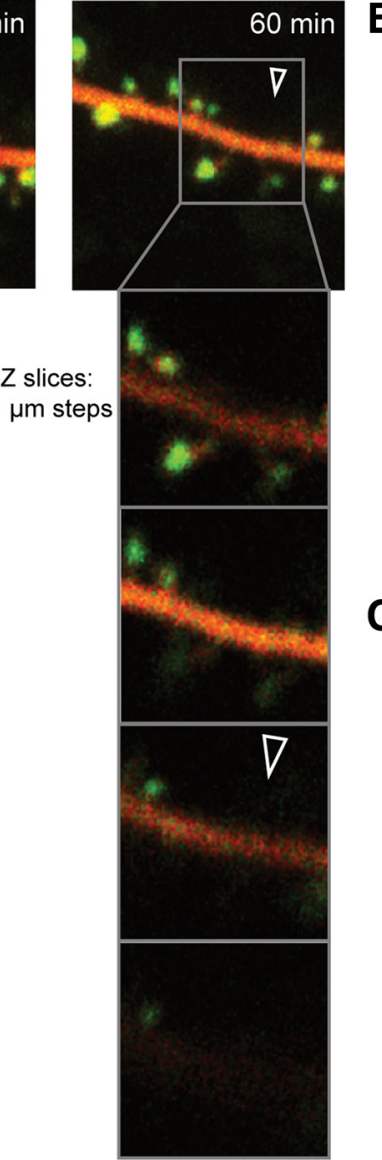

Figure 1. Rates of spine elimination are unaltered in neurons expressing low levels of PSD-95-GFP. A, Images of a dendrite from a hippocampal neuron coexpressing PSD-95-GFP (green) and DsRed-Express (red) at 0 min (left panels) and at 60 min (right spines, 12 cells) and those expressing DsRed-Express alone (blackbars; $n=580$ spines, 11 cells). C, Spine density ( $p=0.8$ ) was not significantly different between cells expressing both PSD-95-GFP and DsRed-Express (green bar) and those expressing DsRedExpress alone (black bar). Error bars indicate SEM.

sities from images of cells expressing GFP alone or DsRed-Express alone, was subtracted from all images before intensity measurements were made. Background subtraction for dendrites was performed by subtracting "distant background" (mean pixel intensity from an ROI distant from the labeled dendrites multiplied by the number of pixels in the dendrite ROI) from the integrated dendritic ROI intensity. To account for higher background fluorescence near the dendritic shaft, background subtraction for spines was performed by subtracting "adjacent background" (integrated pixel intensity from a neighboring ROI of equal dimensions proximal to the dendritic shaft) from the integrated spine ROI intensity. Finally, to account for fluctuations in laser power between time points, bleed-through-corrected and background-subtracted spine intensity measurements were normalized to a dendrite intensity factor, which consisted of the average mean pixel value from three boxed ROIs $\left(\sim 0.3 \mu \mathrm{m}^{2}\right)$ on the associated dendrite in the red (DsRed-Express) channel.

Criterion for spine elimination. Spines were considered to have eliminated when the spine red (DsRed-Express) fluorescence intensity fell within 2 SDs of the background levels near the dendrite. Background levels near the dendrite were calculated by measuring the mean and SD of the "spine" intensity values (as described above, except ROIs were chosen that did not contain a spine) for three ROIs neighboring the eliminated spine and adjacent to the dendrite. On the graph shown, 2 SDs above and below the mean background red fluorescence near the dendrite are plot- 
A
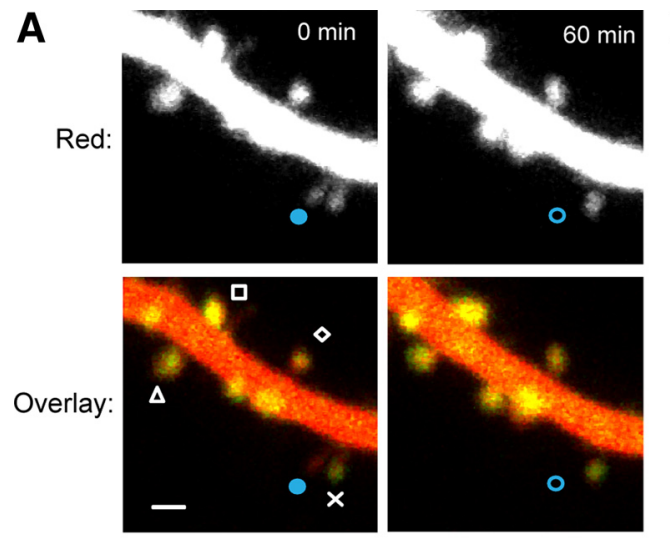

C

$$
\text { Eliminating spines }
$$

- Neighboring spines

口Smallest neighboring spines

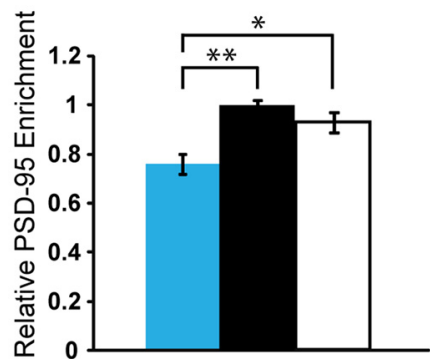

B

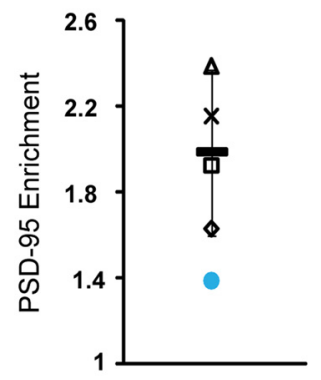

D

Eliminating spines

- Neighboring spines

口Smallest neighboring spines

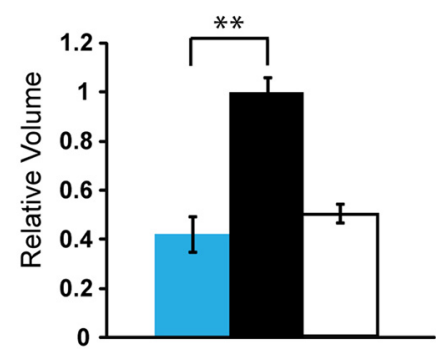

Figure 2. Eliminating spines are less enriched for PSD-95-GFP than persistent neighbors. A, A spine with low levels of PSD-95GFP at 0 min (filled blue circle) eliminated within 60 min (open blue circle), whereas neighboring spines persisted (white symbols). Scale bar, $1 \mu \mathrm{m}$. B, At $0 \mathrm{~min}$, the eliminating spine was less enriched for PSD-95-GFP than its neighbors. The symbols correspond to the spines identified in $\boldsymbol{A}$. The solid bar represents the mean, and error bars represent the SD of the PSD-95-GFP enrichment for the four neighboring spines. C, Eliminating spines (blue bar; $n=37$ spines; 15 cells) were less enriched for PSD-95-GFP than neighboring persistent spines (black bar; $n=96$ spines; $p<0.001$ ) and smallest neighboring persistent spines (white bar; $n=$ 23 spines; $p<0.05$ ). $\boldsymbol{D}$, Eliminating spines (blue bar) were smaller than neighboring persistent spines (black bar; $p<0.001$ ), but they were not smaller than the smallest neighboring persistent spines (white bar; $p=0.4$ ). ${ }^{*} p<0.05 ;{ }^{* *} p<0.001$.

ted as dashed lines. A spine was deemed to have eliminated at the time point at which the integrated spine fluorescence intensity value fell within this background variability.

Quantification of PSD-95-GFP enrichment. PSD-95-GFP enrichment in dendritic spines was calculated from bleed-through-corrected and background-subtracted green (PSD-95-GFP) and red (DsRed-Express) fluorescence intensities (as described above) by dividing the green signal in the spine by the green signal in the dendrite $(\mathrm{Gs} / \mathrm{Gd})$ and normalizing to the value of the red signal in the spine divided by the red signal in the dendrite (Rs/Rd). Relative PSD-95-GFP enrichment in spines was calculated by dividing the enrichment value for each individual spine by the mean enrichment value for four neighboring persistent spines associated with the same dendritic segment. Fold enrichment of PSD-95-GFP in dendritic shafts following spine retraction was calculated from bleedthrough-corrected and background-subtracted green (PSD-95-GFP) and red (DsRed-Express) signal intensities in the dendrite at the site of spine elimination by dividing the green signal by the red signal following spine elimination $\left(\mathrm{Gd}_{t 2} / \mathrm{Rd}_{t 2}\right)$ and normalizing to the green signal divided by the red signal at the first time point imaged $\left(\mathrm{Gd}_{t 1} / \mathrm{Rd}_{t 1}\right)$.

Estimation of relative spine volume and spine length/width ratio. Relative spine volume was estimated from bleed-through-corrected and background-subtracted red (DsRed-Express) fluorescence intensities by dividing the integrated spine intensity value by the mean integrated spine intensity value of four neighboring spines on the same dendrite. Spine brightness measurements give an accurate estimate of relative spine volume when compared with electron microscopy (Holtmaat et al., 2005). Spine length/width ratio was defined as the ratio of the length from the tip of the spine head to the spine neck base (spine length) to the width across the spine head at its widest point (spine width) or, if the widest point was ambiguous, at the maximum dimension perpendicular to spine length.

Statistics. Error bars represent SEM (unless otherwise noted), and significance was set at $p=0.05$ (two-tailed $t$ test). The single asterisks indicate $p<0.05$, and the double asterisks indicate $p<0.001$. $r$ is the correlation coefficient.

\section{Results \\ Eliminating spines are overall less enriched for PSD-95-GFP}

To examine the relationship between dendritic spine stability and PSD-95 content, we monitored spine morphology (DsRedExpress) and PSD-95 levels (PSD-95GFP) over time using dual-color time-lapse two-photon microscopy. Because high levels of PSD-95-GFP expression can alter spine morphology and density (ElHusseini et al., 2000), we first optimized expression levels of DsRed-Express and PSD-95-GFP. Hippocampal pyramidal neurons in organotypic slice cultures from neonatal rats were cotransfected at DIV5DIV6 with DsRed-Express (10 $\mu \mathrm{g})$ and PSD-95-GFP $(2,4$, or $10 \mu \mathrm{g})$. Dendrites of transfected neurons were imaged either 1 or $2 \mathrm{~d}$ after transfection, and spine densities, lengths, and turnover rates (gains and losses) were quantified. Spine gains and losses were confirmed by examining individual $1 \mu \mathrm{m}$ optical $Z$ sections spanning the dendritic segment (Fig. $1 A$ ). At optimal conditions ( $4 \mu \mathrm{g}$ of PSD-95-GFP, $1 \mathrm{~d}$ after transfection), spine morphology and PSD-95-GFP levels could be readily visualized and spine densities and turnover rates did not significantly differ between cells expressing both DsRed-Express and PSD-95-GFP and those expressing DsRed-Express alone (Fig. $1 B, C$ ).

To quantitatively determine whether eliminating spines contain lower levels of PSD-95-GFP than their neighbors, we monitored PSD-95-GFP and DsRed-Express levels at the first imaging time point in spines that retracted within $1 \mathrm{~h}$ and in neighboring spines that persisted (Fig. 2A,B). Because PSD-95 content is correlated with PSD size (Aoki et al., 2001) and PSD size is correlated with spine size (Harris and Stevens, 1989), larger spines would be expected to have a higher PSD-95-GFP content. Therefore, we quantified PSD-95-GFP enrichment by normalizing PSD-95GFP content to spine volume using DsRed-Express measurements. We found that spines that retracted were on average relatively less enriched for PSD-95-GFP $(0.76 \pm 0.04)$ than their persistent neighbors $(1.0 \pm 0.02 ; p<0.001$; Fig. $2 C)$. Notably, eliminating spines were also smaller in volume $(0.42 \pm 0.07)$ than their persistent neighbors $(1.0 \pm 0.06$; $p<0.001$; Fig. $2 D)$. To examine whether differences in spine volume contribute to differences in PSD-95-GFP enrichment in eliminating versus persistent spines, we compared PSD-95-GFP enrichment in eliminating spines to PSD-95-GFP enrichment in the smallest neighboring persistent spines. Eliminating spines were still significantly less enriched for PSD-95-GFP than these smallest neighbors $(0.92 \pm 0.04 ; p<0.05$; Fig. $2 C)$, even though these smallest persistent neighbors were similarly sized to eliminating 
spines $(0.50 \pm 0.04 ; p=0.4 ;$ Fig. $2 D)$. Therefore, we conclude that eliminating spines are less enriched for PSD-95-GFP than their persistent neighbors and that this decreased PSD-95-GFP enrichment is not simply because eliminating spines are smaller.

\section{A substantial subset of eliminating spines are enriched for PSD-95-GFP}

Spines that are enriched for PSD-95-GFP are almost always juxtaposed to presynaptic markers, suggesting that these spines are incorporated into synapses (Prange and Murphy, 2001). Therefore, the finding that eliminating spines are overall less enriched in PSD-95-GFP may be due to the fact that eliminating spines are comprised predominantly of transient spines that are not engaged in synapses. However, spine elimination is thought to contribute to circuit refinement during development and to circuit modifications during synaptic plasticity in adults (Xu et al., 2009; Yang et al., 2009; Roberts et al., 2010), suggesting that, at least in some cases, spine elimination must be accompanied by modifications in synaptic connectivity. For this reason, we expected that a subset of eliminating spines would initially be engaged in synapses and also enriched for PSD-95-GFP. Indeed, we often observed that retracting spines were as enriched for PSD-95-GFP as their persistent neighbors (Fig. $3 A, B$ ).

To estimate the fraction of eliminating spines that were initially either (1) less enriched or (2) comparably enriched for PSD95-GFP as their persistent neighbors, we divided spines that eliminated into two groups based upon their deviation from the mean PSD-95-GFP enrichment of four neighboring persistent spines on the same dendrite (Fig. $3 C$ ). Eliminating spines with PSD-95-GFP enrichment values at the first imaging time point that were $<1$ SD lower than the mean PSD-95-GFP enrichment of four persistent neighbors were considered relatively unenriched and classified as "type I." Eliminating spines with relative enrichment values at the first imaging time point that were within $1 \mathrm{SD}$ of, or $>1 \mathrm{SD}$ higher than, the mean enrichment of four persistent neighbors were considered comparably enriched and classified as "type II." The cutoff of 1 SD lower than the mean was optimal for matching type I and type II categories most closely to spines classified qualitatively as "unenriched" and "comparably enriched" for PSD-95-GFP compared with their persistent neighbors. Consistent with eliminating spines being on average less enriched for PSD-95-GFP than their persistent neighbors (Fig. 2), the distribution of relative PSD-95-GFP enrichment values for eliminating spines was strongly shifted toward less enriched values, compared with the distribution for control spines (Fig. 3C).

We examined the percentage of total spine eliminations attributed to spines that were initially classified as type I versus type II at the first imaging time point (Fig. 3D). As expected, we found that a majority $(62 \%)$ of spines that retracted within $1 \mathrm{~h}$ were classified as type I, or relatively unenriched for PSD-95-GFP compared with their persistent neighbors, suggesting that a majority of retracting spines may not be engaged in synapses. Yet a significant percentage (38\%) of spines that retracted within $1 \mathrm{~h}$ were classified as type II, or comparably enriched for PSD-95GFP as their persistent neighbors, suggesting that a large subset of retracting spines might initially have been engaged in synapses. Notably, if we limited our analysis to the subset of spines that retracted within $15 \mathrm{~min}$ of the first imaging time point, we found that the overwhelmingly majority (79\%) of retracting spines were classified as type I, or relatively unenriched for PSD-95-GFP compared with their persistent neighbors, and only a small frac-
A
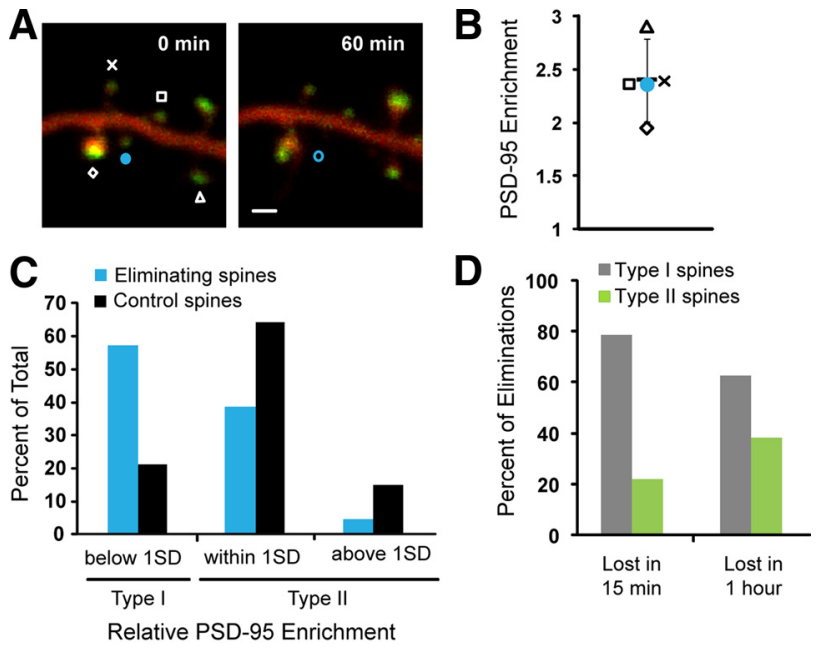

E

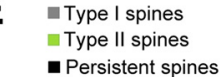

F $\quad$ Type I spines $\square$ Type II spines - Persistent spines
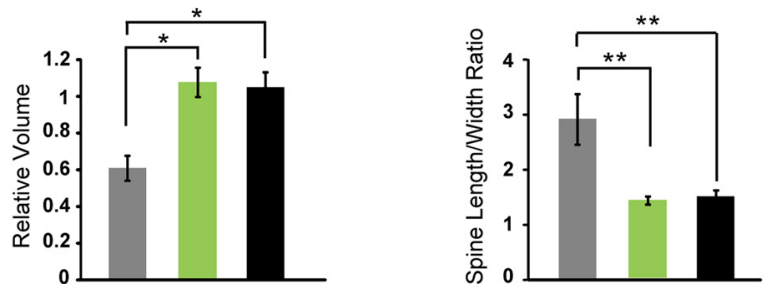

Figure 3. A subset of eliminating spines are as enriched for PSD-95-GFP as their persistent neighbors. A, A spine enriched for PSD-95-GFP at 0 min (filled blue circle) eliminated within 60 $\min$ (open blue circle). Scale bar, $1 \mu \mathrm{m}$. $\boldsymbol{B}$, At 0 min, the eliminating spine was as enriched for PSD-95-GFP as its neighbors. Symbols correspond to the spines identified in $\boldsymbol{A}$. The solid bar represents the mean, and error bars represent the SD of the PSD-95-GFP enrichment for the four neighboring spines. $C$, Percentage of eliminating spines (blue bars; $n=44$ ) or control spines (chosen blindly with regards to their fate; black bars; $n=81$ ) that were $<1$ SD less enriched for PSD-95-GFP (type I), within 1 SD, or >1 SD more enriched for PSD-95-GFP (type II) than the mean of four neighboring spines. $\boldsymbol{D}$, Percentage of spines that eliminated within $15 \mathrm{~min}$ ( $n=$ 14) or $60 \mathrm{~min}$ ( $n=37$ ) that were classified as type I (gray bars) or type II (green bars) at $0 \mathrm{~min}$. $\boldsymbol{E}$, Type I spines (gray bar; $n=17$ ) were significantly smaller than type II spines (green bar; $n=$ 83; $p<0.05$ ) or persistent spines (black bar; $n=84 ; p<0.05$ ). F, Type I spines (gray bar; $n=$ 17) had a significantly higher spine length/width ratio than that of type II spines (green bar; $n=83 ; p<0.01$ ) or persistent spines (black bar; $n=84 ; p<0.01$ ). ${ }^{*} p<0.05 ;{ }^{* *} p<0.001$.

tion $(21 \%)$ were classified as type II, or comparably enriched for PSD-95-GFP.

To investigate whether type I and type II spines represent distinct populations of spines as defined by parameters beyond their relative PSD-95-GFP enrichment values, we compared the two spine types using additional parameters. We examined all type I and type II spines, regardless of whether they eliminated. We found that type I spines $(0.61 \pm 0.07)$ were significantly smaller than type II spines $(1.08 \pm 0.08 ; p<0.05$; Fig. $3 E)$ and that type I spines $(2.92 \pm 0.46)$ were significantly longer and thinner than type II spines $(1.41 \pm 0.18 ; p<0.001$; Fig. $3 F)$. Therefore, we conclude that type I and type II spines comprise different populations, with type I spines being relatively unenriched for PSD-95-GFP, smaller in volume, and more filipodialike compared with type II spines.

\section{Loss of PSD-95-GFP enrichment is not a prerequisite for spine retraction}

Our finding that $79 \%$ of spines that retracted within $15 \mathrm{~min}$, compared with only $62 \%$ of spines that retracted within $1 \mathrm{~h}$, were relatively unenriched for PSD-95-GFP supports the possibility 
A
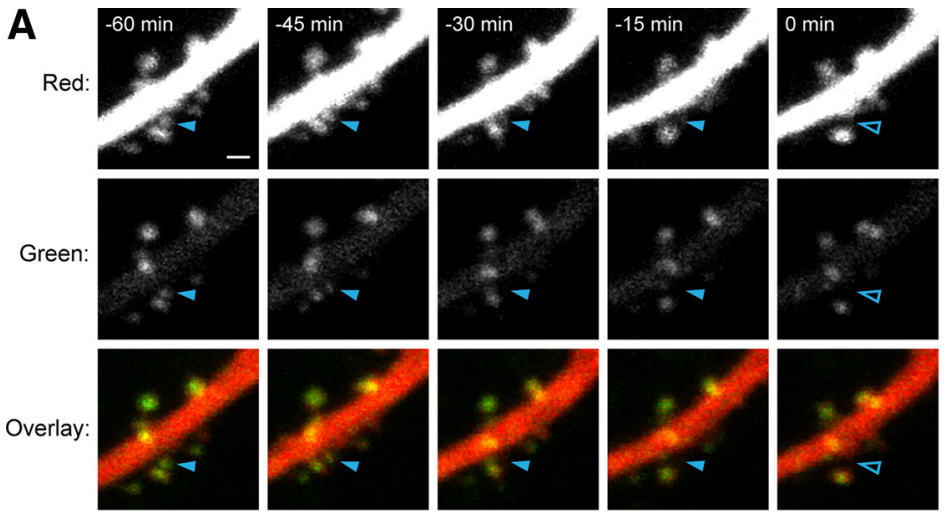

\section{B}
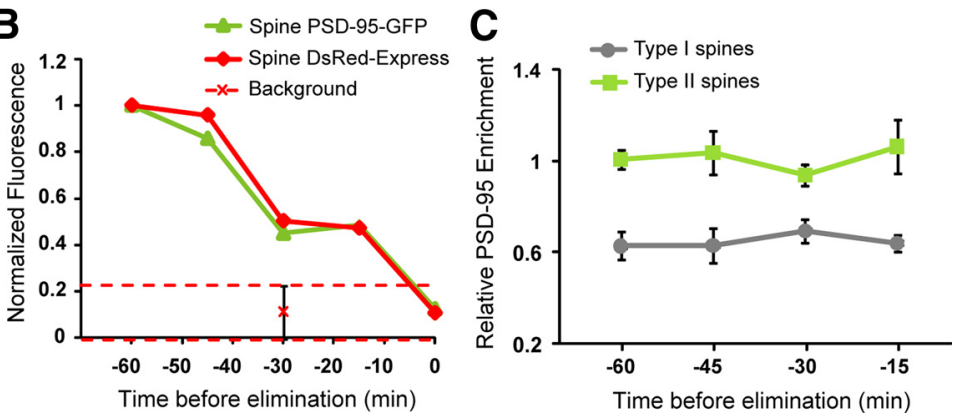

Figure 4. Loss of PSD-95-GFP enrichment is not a prerequisite for spine retraction. A, A type II spine (filled blue arrowhead) eliminated (open blue arrowhead) during time-lapse imaging of a dendrite from a hippocampal neuron coexpressing PSD-95-GFP (green) and DsRed-Express (red). Time stamps are relative to time of spine elimination. Scale bar, $1 \mu \mathrm{m}$. $\boldsymbol{B}$, Red (red diamonds) and green (green triangles) fluorescence intensity of the eliminating spine in $\boldsymbol{A}$ normalized to the first time point and plotted relative to time of spine elimination. Mean (red cross) and 2SDs above and below the mean (red dashed lines) background red fluorescence intensity near the dendrite at time 0 are also plotted. C, Relative enrichment for PSD-95-GFP (compared with the mean of four persistent neighboring spines) in eliminating type I (gray circles; $n=25$ ) or type II (green squares; $n=18$ ) spines did not change during spine elimination. Eliminating type II spines were significantly more enriched for PSD-95-GFP than eliminating type I spines at all time points $(p<0.001)$. Error bars indicate SEM.

that eliminating type II spines (initially comparably enriched for PSD-95-GFP as persistent neighbors) pass through a stage in which PSD-95-GFP enrichment is significantly reduced before retraction. To examine the temporal relationship between spine retraction and PSD-95-GFP enrichment levels, we examined PSD-95-GFP enrichment in eliminating spines relative to persistent neighbors at $15 \mathrm{~min}$ intervals leading up to spine elimination (Fig. 4A). A spine was considered to have eliminated when its red (DsRed-Express) fluorescence intensity fell within 2 SDs of the mean background fluorescence level adjacent to the dendrite. We found that fluctuations in green (PSD-95-GFP) and red (DsRedExpress) fluorescence intensity values in individual spines (Fig. $4 B$ ) were highly correlated during spine retraction $(r=0.79$; $p<$ 0.001 , partial correlation analysis; $n=17$ spines, 11 cells), and therefore the enrichment of PSD-95-GFP was stable over time. There was no evidence for a systematic decrease in PSD-95-GFP enrichment in eliminating type II spines down to levels comparable with that of eliminating type I spines, even at the time point immediately before spine retraction (Fig. 4C), suggesting that spines can destabilize and retract even while retaining PSD-95GFP enrichment levels comparable with neighboring persistent spines.

Changes in PSD-95-GFP fluorescence were not only closely matched to changes in DsRed-Express fluorescence in spines that eliminated, but this was also true for spines that persisted for the imaging session. Decreases and increases in PSD-95-GFP and DsRed-Express fluorescence were highly correlated in persistent neighbors during 15 min time-lapse intervals $(r=0.81 ; p<$
0.001, partial correlation analysis; $n=44$ spines, 11 cells), leading to relatively stable PSD-95-GFP enrichment levels in individual spines. To determine whether faster resolution time-lapse would reveal that changes in spine volume were routinely preceded by changes in PSD-95GFP enrichment, we monitored spine DsRed-Express and PSD-95-GFP fluorescence from neurons that were imaged at $20 \mathrm{~s}$ intervals for a total of $5 \mathrm{~min}$ (Fig. $5 A, B)$. Again at this higher temporal resolution, PSD-95-GFP levels and relative spine volume were tightly coupled $(r=$ $0.68 ; p<0.001$, partial correlation analysis; $n=43$ spines, 6 cells). Shifting PSD-95-GFP fluorescence measurements earlier or later with respect to DsRed-Express fluorescence measurements (Fig. 5C) in all cases led to decreased correlation. We found no evidence that the PSD-95-GFP fluorescence changes preceded spine volume changes even at this high temporal resolution timelapse.

Because PSD-95-GFP enrichment is maintained during spine retraction, when spines shrink there is a corresponding decrease in absolute levels of PSD-95-GFP. It is possible that there is a minimum level of PSD-95-GFP that is required to maintain a spine, and that any spine below this critical level would become unstable and retract. However, we think that this is unlikely. First, we found no significant difference in the PSD-95-GFP fluorescence intensities (normalized to dendritic fluorescence to compensate for variations in laser power between different preparations) of type I spines that eliminated ( $216 \pm 63$ A.U.; $n=10$ spines $)$ compared with type I spines that persisted ( $249 \pm 62$ A.U.; $n=7$ spines; $p=0.72$ ). Second, we found no significant difference in PSD-95-GFP enrichment level between type I spines that persisted ( $0.52 \pm 0.10 ; n=7$ spines $)$ and type I spines that eliminated within an hour $(0.51 \pm 0.048 ; n=10$ spines; $p=0.72)$. Third, we found that type I spines with the lowest relative PSD-95-GFP enrichment levels and the smallest relative volumes, and by inference the lowest absolute PSD-95-GFP levels, could persist for entire imaging sessions (up to $2 \mathrm{~h} ; n=2$ spines). Furthermore, when we restricted our analysis to type II spines, we found that $11 \%$ of spines that persisted for the entire imaging session had absolute PSD-95-GFP levels lower than the mean absolute PSD95-GFP value of type II spines that eliminated. These data do not support the hypothesis that there is a critical level of PSD-95 that is required for spine survival.

PSD-95-GFP is often enriched in the dendritic shaft at the site of spine elimination

Despite our observation that loss of PSD-95-GFP from type II spines was tightly coupled to the loss of spine volume, we often observed an enrichment of PSD-95-GFP on the dendrite at the site of spine elimination (Fig. 6A), suggesting that PSD-95-GFP did not always completely disperse when spines retracted. To determine whether PSD-95-GFP became significantly enriched on the dendrite following spine elimination, we quantified the 
fold enrichment of PSD-95-GFP in the shaft at the site of spine elimination and at the base of neighboring spines (Fig. 6B). Overall, we found no significant difference in PSD-95-GFP enrichment at the base of newly eliminated spines (1.06 \pm $0.05)$ compared with the base of persistent neighbor spines $(0.99 \pm 0.03 ; p=0.3$; Fig. $6 C)$. However, $33 \%$ of the type II spine eliminations were associated with an increase in PSD-95-GFP enrichment at the base that was $>2$ SDs more than the mean relative enrichment of neighbors; we classified these as "type IIb" spines (Fig. 6D). Finding such a large PSD-95-GFP fold enrichment at the base following a type I spine elimination was a rare occurrence (1 of 25; Fig. 6D), strongly suggesting that the PSD-95-GFP enrichment at the base of type IIb spines originated from PSD95-GFP that was originally in the spine head. Consistent with the idea that this subset of spines was becoming enriched for PSD-95-GFP before retracting, there was a trend toward increased PSD-95GFP enrichment in type IIb spines at the time point immediately before elimination (Fig. $6 E$ ). While the remaining $67 \%$ of type II ("type IIa") spines did not exhibit a strong fold enrichment at their base, 8 of 13 of these spines did have a diffuse remnant of PSD-95-GFP on the dendritic shaft at the site of spine retraction.

To examine whether the enhanced enrichment for PSD-95-GFP at the base of newly eliminated type IIb spines was transient, or long-lasting, we quantified PSD95-GFP enrichment at the base in additional time points after retraction. We found that fold enrichment in the shaft at the base of type IIb spines remained significantly elevated relative to that of neighboring spines for the remainder of the imaging session (15-60 min after elimination; Fig. 7A,B). In addition, the red signal from a region of the dendritic shaft at the base of these eliminated type IIb spines compared with the surrounding dendrite $(1.28 \pm 0.09)$ was significantly higher than the red signal at the base of neighboring spines compared with the surrounding dendrite $(0.98 \pm 0.05 ; p<0.05$; Fig. $7 C)$. These data suggest that the persistent PSD-95-GFP enrichment at the base of eliminated type IIb spines might be due to persistent subresolution spines in the $\mathrm{Z}$ direction. Alternatively, the remaining PSD-95-GFP enrichment could reflect a partially intact PSD, an orphan PSD without a presynaptic partner, or the conversion of a spine synapse to a shaft synapse. Notably, the red signal associated with immobile and persistent shaft PSD-95-GFP puncta, which we speculated were engaged in shaft synapses, was also significantly larger than the nearest surrounding dendrite $(1.25 \pm 0.06 ; p=0.8$; Fig. $7 C)$.

\section{NMDA receptor inhibition does not alter PSD-95-GFP} enrichment during spine elimination

NMDA receptor (NMDAR) inhibition decreases spine elimination rates by more than twofold (Yasumatsu et al., 2008). We wondered whether inhibition of NMDARs influences spine elimination rates

B
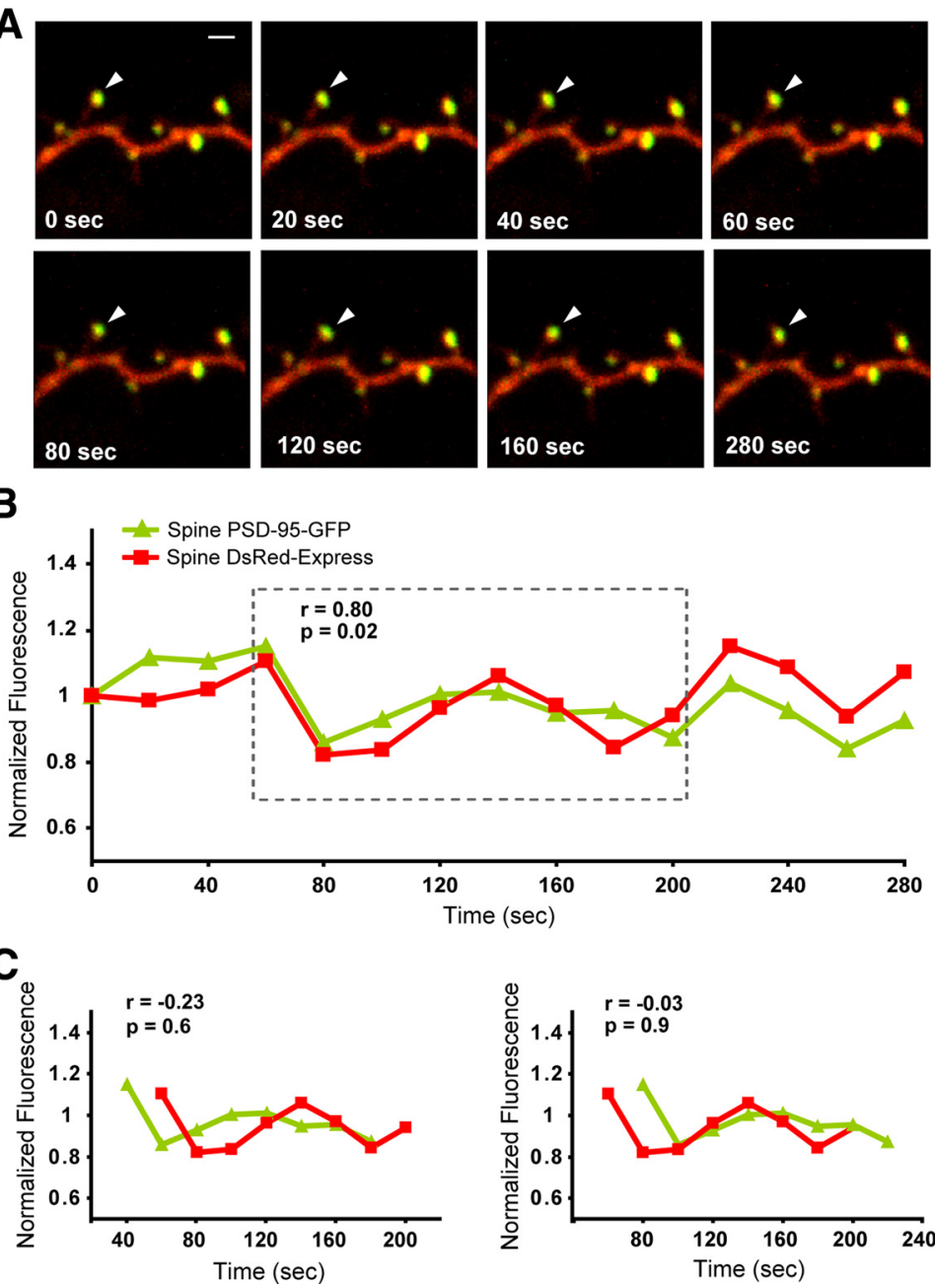

Figure 5. Changes in PSD-95-GFP and spine volume are tightly coupled in persistent spines. $A$, Images of a dendrite from a hippocampal neuron coexpressing PSD-95-GFP and DsRed-Express imaged at 20 s intervals. Scale bar, $1 \mu \mathrm{m}$. $\boldsymbol{B}$, Normalized red (red squares) and (green triangles) fluorescence intensity of the spine identified in $\boldsymbol{A}$ (white arrowhead) were highly correlated during rapid time-lapse. $C$, No significant correlation was detected in red and green fluorescence when green fluorescence was shifted with respect to red fluorescence 20 s earlier (left panel) or 20 s later (right panel) than the time of actual measurements (same data as in dotted box in $\boldsymbol{B}$ ).

by altering PSD-95-GFP enrichment levels during spine retraction. Therefore, we examined PSD-95-GFP enrichment in eliminating spines in the presence of $10 \mu \mathrm{M}$ CPP to inhibit NMDARs (Fig. $8 A$ ). We found that in the presence of CPP, as under control conditions, fluctuations in DsRed-Express and PSD-95-GFP fluorescence intensity values (Fig. $8 \mathrm{~B}$ ) were highly correlated during spine retraction $(r=0.88 ; p<0.001$, partial correlation analysis; $n=6$ spines, 5 cells). Type II spines in the presence of CPP destabilized and retracted while maintaining PSD-95-GFP enrichment levels in a manner not significantly different from that observed under control conditions (Fig. 8C). Indeed, small remnants of PSD-95-GFP were often observed on the dendritic shaft at the site of spine retraction, and in one case the PSD-95-GFP enrichment at the base following spine retraction was substantial enough that the eliminating spine quantified as a type IIb (Fig. 8A). We found no detectable difference in the relationship between PSD-95-GFP enrichment and spine volume during spine elimination in the presence of CPP.

\section{Discussion}

PSD-95 enrichment and spine destabilization

To quantify the relationship between PSD-95 enrichment and spine stability, we simultaneously monitored dendritic spine 
A
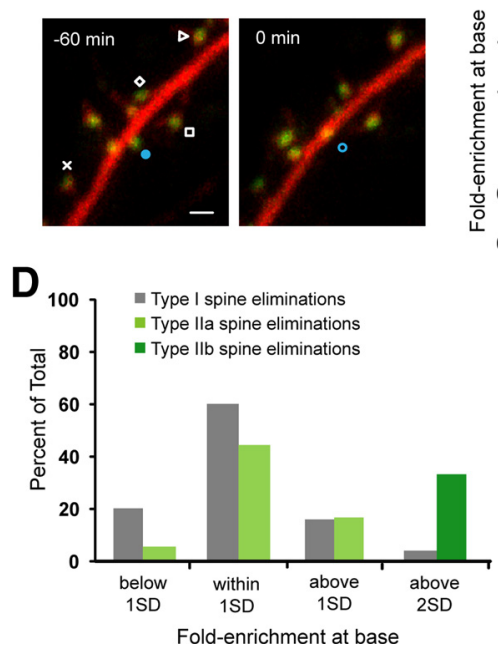

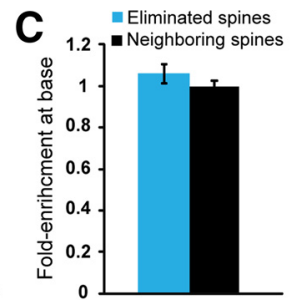

E

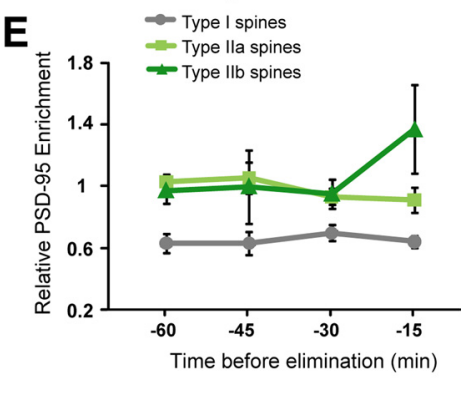

Figure 6. PSD-95-GFP is often enriched in the dendritic shaft at the site of spine elimination. $\boldsymbol{A}$, The dendritic shaft was enriched for PSD-95-GFP (open blue circle) at the site of elimination of a type II spine (solid blue circle). Scale bar, $1 \mu \mathrm{m}$. $\boldsymbol{B}$, Immediately following spine elimination, fold enrichment (relative to the same site 60 min prior) of PSD-95-GFP on the dendritic shaft at the base of the eliminated spine was higher than at the base of neighboring persistent spines. The symbols correspond to the spines identified in $\boldsymbol{A}$. The solid bar represents the mean and error bars represent two times the SD of the fold enrichment of PSD-95-GFP at the base of four neighboring persistent spines. C, Fold enrichment of PSD-95-GFP at the base of eliminated spines (blue bar; $n=$ 44) was not significantly different from that at the base of neighboring persistent spines (black bar; $n=35 ; p=0.3$ ). $\boldsymbol{D}$, Percentage of type I (gray bars; $n=25$ ) and type II (green bars; $n=18$ ) spine eliminations that were associated with a fold enrichment of PSD-95-GFP on the dendritic shaft at the site of spine retraction that was below, within, or above 1 or 2 SD of the mean fold enrichment of PSD-95-GFP at the base of four persistent neighboring spines. One-third of type II spines (type Ilb; dark green bar) showed a fold enrichment of PSD-95-GFP on the dendritic shaft following elimination that was $>2$ SD more than the mean fold enrichment at the base of four persistent neighbors, whereas only 1 of 25 type I spines showed such a strong fold enrichment. $\boldsymbol{E}$, Eliminating type Ila (light green squares; $n=12$ ) spines were significantly more enriched for PSD-95-GFP than eliminating type I spines (gray circles; $n=25$ ) at all time points $(p<0.01)$. Eliminating type Ilb (dark green triangles; $n=6$ ) spines showed a trend toward increased enrichment of PSD-95-GFP at the time point immediately before spine retraction.

morphology and PSD-95-GFP levels in dendrites of developing hippocampal pyramidal neurons. Although others have studied the dynamics of PSD-95-GFP in living neurons (Okabe et al., 1999, 2001; Marrs et al., 2001; Prange and Murphy, 2001; De Roo et al., 2008), those studies have focused on PSD-95-GFP content and clustering; none have quantified PSD-95-GFP enrichment over time. Our results demonstrate that the majority $(62 \%)$ of spines that retracted within an hour were relatively less enriched for PSD-95-GFP (type I) than their persistent neighbors, consistent with previous reports that spines with low levels of PSD-95 are more dynamic (Prange and Murphy, 2001; Ehrlich et al., 2007; De Roo et al., 2008). However, more than one-third (38\%) of spines that retracted within an hour were as enriched for PSD95-GFP (type II) as their persistent neighbors. We hypothesized that these type II spines might pass through a step before eliminating in which they experienced a dramatic decrease in enrichment for PSD-95-GFP down to levels comparable with that of type I spines. Contrary to our hypothesis, we did not detect a loss of PSD-95-GFP enrichment in eliminating type II spines, not even at the time point immediately before spine loss, suggesting that spine destabilization is not regulated by PSD-95 enrichment levels.

It is important to consider that we have used PSD-95-GFP to infer the behavior of endogenous PSD-95 during spine elimination. Although we were careful to express PSD-95-GFP at low levels that did not induce the elevated spine densities associated with high levels of PSD-95-GFP expression (Craven et al., 1999; El-Husseini et al., 2000), we cannot exclude the possibility that even these low levels of PSD-95-GFP increased enrichment of
PSD-95 in dendritic spines. However, we think it unlikely that our main findings are artifacts of PSD-95-GFP overexpression for multiple reasons. First, we observed a broad range of spine PSD95-GFP enrichment levels, suggesting that we had not swamped all individual spines with excessive levels of PSD-95-GFP. Second, driving excess PSD-95-GFP into previously unenriched spines would likely have resulted in increased spine stability (De Roo et al., 2008); however, we observed no change in spine elimination rates. Finally, because we were able to observe transient type I spines with minimal levels of PSD-95-GFP, we feel confident that if type II spines had transitioned to lower enrichment levels, comparable with those of type I spines, we would have been able to detect this transition.

Our results are surprising in the context of experiments strongly supporting that PSD-95 enrichment regulates spine stability during new spine formation. Contrary to the tight coupling of PSD-95GFP levels and spine volume that we observed during spine elimination, spine outgrowth has been shown to precede PSD-95-GFP accumulation with a time delay on the order of hours (Okabe et al., 2001; De Roo et al., 2008). Arrival of PSD-95 is associated with new spine stabilization, and inhibiting neuronal activ-

ity inhibits both new spine stabilization and the percentage of new spines containing PSD-95-GFP (De Roo et al., 2008), suggesting that enrichment of PSD-95 in new spines plays a key role in regulating their stability. In contrast, we found that spines shrink and retract while maintaining PSD-95GFP enrichment levels comparable with those of persistent neighbors, suggesting that once PSD-95 enrichment is established, it is by itself insufficient to ensure long-term spine stability. Our data support a role for PSD-95 in spine destabilization and retraction that is mechanistically distinct from its role during spine formation and stabilization.

That we did not detect a loss of PSD-95-GFP enrichment before spine shrinkage suggests that spine retraction may be governed by a mechanism mediating parallel and simultaneous decrease of PSD-95 content and spine volume. Indeed, we found that changes in spine volume and PSD-95-GFP levels were tightly correlated both in retracting spines and in persistent neighboring spines. This coupling could potentially be explained by the fact that the actin cytoskeleton interacts with the PSD and also maintains spine shape (Sekino et al., 2007; Blanpied et al., 2008). Furthermore, many regulators of the actin cytoskeleton have been shown to interact with PSD-95 (Pak et al., 2001; Penzes et al., 2001; Lee et al., 2006). Alternatively, activity-dependent mechanisms could lead to independent, but coordinated changes in spine volume and synapse size (Matsuzaki et al., 2004; Zhou et al., 2004; Kopec et al., 2006). In fact, long-term depression has been associated with internalization of PSD components, including PSD-95 and AMPA-type glutamate receptors, via endocytosis (Fitzjohn et al., 2001; Hsieh et al., 2006; Bhattacharyya et al., 2009; Han et 
A
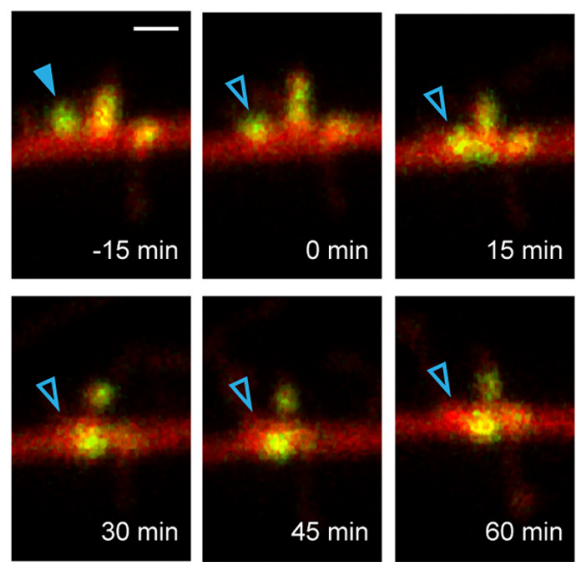

B

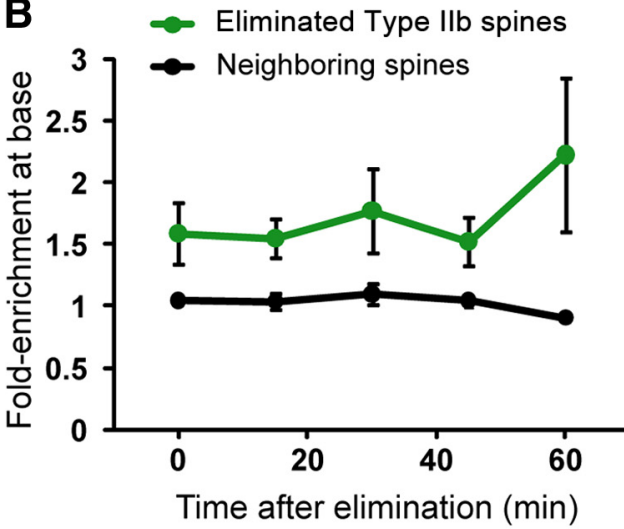

C

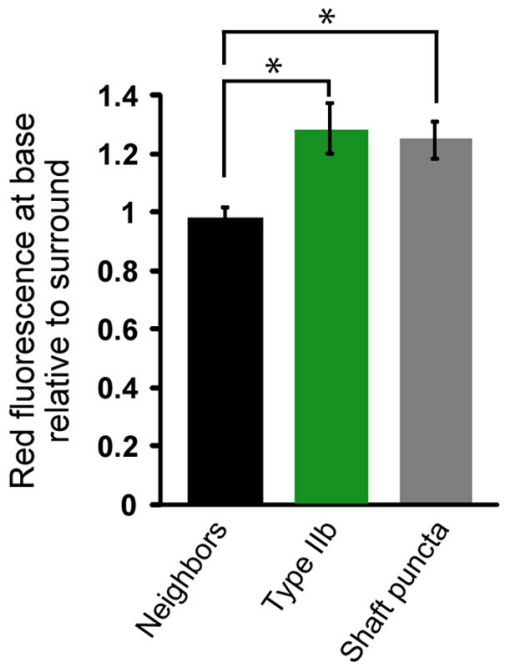

Figure 7. Enrichment of PSD-95-GFP at the site of spine retraction is persistent. $\boldsymbol{A}$, Enrichment of PSD-95-GFP (open blue arrowhead) persisted on the dendritic shaft following elimination of a type llb spine (filled blue arrowhead). Scale bar, $1 \mu \mathrm{m}$. $\boldsymbol{B}$, Enrichment of PSD-95-GFP on the dendritic shaft at the site of eliminated type Ilb spines (green circles; $n=6$ ) was persistently higher than that on the shaft at the site of neighboring spines (black circles; $n=20 ; p<0.05$ at all time points). $C$, Red fluorescence from an ROI on the dendritic shaft at the site of eliminated type Ilb spines compared with the nearest surrounding dendrite (green bar; $n=6$ ) was significantly higher than that at the base of neighboring persistent spines (black bar; $n=6 ; p<$ 0.05 ), but no different from that of persistent and immobile shaft PSD-95-GFP puncta (gray bar; $n=6 ; p=0.8)$. Error bars indicate SEM. ${ }^{*} p<0.05$.

al., 2009). Even independent of neural activity, as in the presence of CPP, similar endocytic mechanisms might be responsible for the coupling of spine shrinkage and loss of PSD-95-GFP during spine retraction.
Relationship between spine loss and synapse loss

Many transient spines likely form and are lost without ever engaging in mature synapses. Because accumulation of PSD-95GFP in new persistent spines occurs over the time course of many hours (De Roo et al., 2008), it is likely that transient spines are unenriched for PSD-95-GFP and therefore fall within our type I category. Supporting this possibility, in our experiments, only $39 \%$ of type I spines survived for $1 \mathrm{~h}$, compared with $93 \%$ of type II spines. However, almost one-half of spines eliminating within $1 \mathrm{~h}$ were type II, or comparably enriched for PSD-95-GFP as their neighbors, suggesting that they were initially engaged in synapses. How is spine loss related to synapse loss in these cases? Our observation that spine volume decreases and loss of PSD-95-GFP were highly correlated for type II spines suggests that, in most cases, spine retraction and postsynaptic disassembly are tightly linked.

We observed that the dendritic shaft sometimes became enriched for PSD-95-GFP at the site of spine elimination. This could be explained by the persistent presence of a small subresolution spine. However, previous comparisons between twophoton imaging and retrospective serial section EM of the same dendritic segments confirm a robust ability to identify all spines with two-photon imaging (Holtmaat et al., 2005; Knott et al., 2006). In addition, our fluorescence measurements indicated that these dendritic PSD-95-GFP enrichments were indistinguishable from long-lived and immotile shaft PSD-95-GFP puncta, suggesting that they might be shaft synapses. In the mature brain, the maintenance of synaptic contacts during spine retraction is unlikely because the neuropil is very entangled (Knott et al., 2006). Indeed, in vivo time-lapse imaging of axons in the adult mouse has revealed that boutons are relatively immobile compared with dendritic spines (De Paola et al., 2006), and therefore it seems unlikely that active zones "chase" retracting PSDs, maintaining functional synapses. However, bouton movement may be less restricted in young in vitro organotypic slices, and it is possible that some synapses remained intact following spine retraction. Alternatively, these dendritic PSD-95-GFP enrichments could represent orphan PSDs without presynaptic partners and, during the period that the interconnected PSD-95 lattice (Blanpied et al., 2008) remains intact, there may exist a window of opportunity for accelerated synapse reformation. U1timately, the precise nature of these shaft puncta will remain speculative until experiments using retrospective EM reveal the dendritic ultrastructure at these sites.

Recent studies suggest that long-term decreases in synaptic strength induced by low-frequency stimulation (LFS) are likely governed in part by the loss of synapses that takes place via both presynaptic and postsynaptic structural rearrangement, including the shrinkage and loss of dendritic spines (Zhou et al., 2004; Bastrikova et al., 2008; Becker et al., 2008). However, the fate of the PSD in such studies has not yet been examined; it remains to be determined how LFS affects PSD-95 enrichment in dendritic spines, and whether activity-dependent changes in PSD stability are integral for structural plasticity in response to LFS. Further examination of how LFS influences presynaptic and postsynaptic morphology, including a more direct assessment of how those structural changes affect synapse composition and function, will ultimately help elucidate the important role of spine synapse elimination in mediating adaptive plasticity of the brain.

\section{References}

Aoki C, Miko I, Oviedo H, Mikeladze-Dvali T, Alexandre L, Sweeney N, Bredt DS (2001) Electron microscopic immunocytochemical detection of 
A
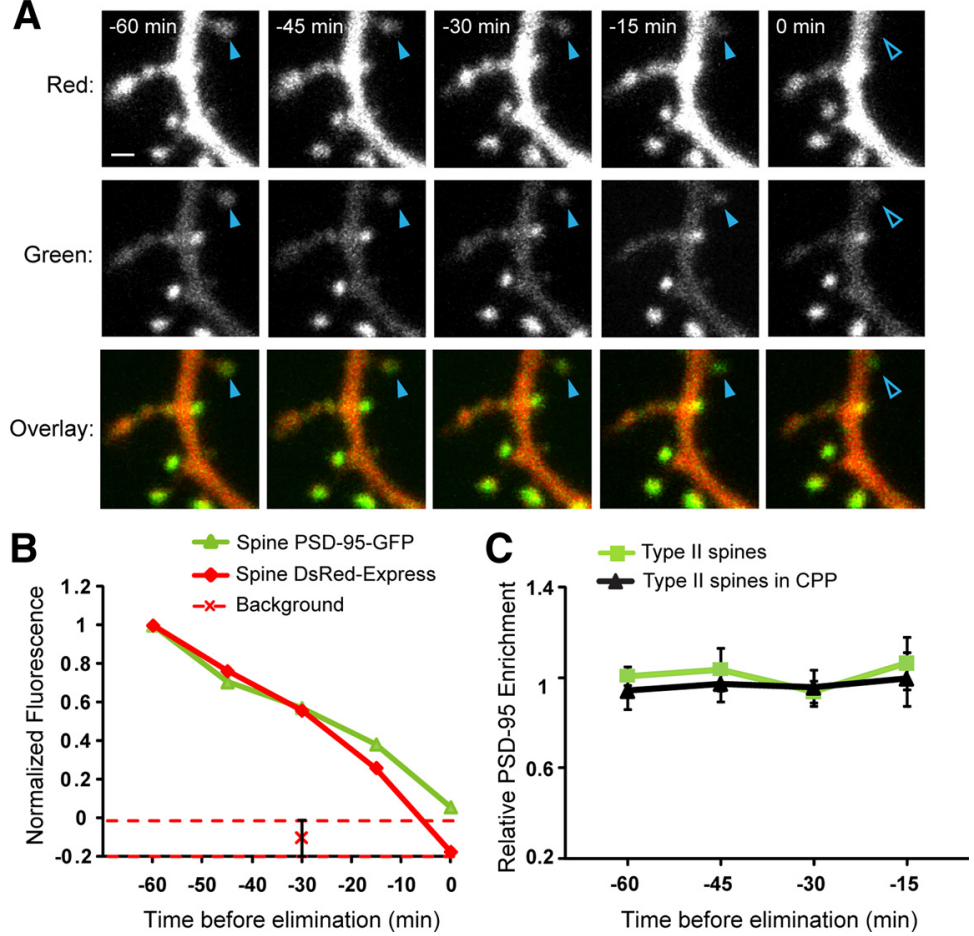

Figure 8. NMDAR inhibition does not alter PSD-95-GFP enrichment during spine elimination. $\boldsymbol{A}, \mathrm{A}$ type II spine (filled blue arrowhead) eliminated (open blue arrowhead) in the presence of $10 \mu \mathrm{M}$ CPP. Consistent with the visible puncta of PSD-95-GFP that remained at the site of spine retraction at $t=0 \mathrm{~min}$ (open blue arrowhead), this example quantified as a type llb spine. Time stamps are relative to time of spine elimination. Scale bar, $1 \mu \mathrm{m}$. $\boldsymbol{B}$, Red (red diamonds) and green (green triangles) fluorescence intensity of the eliminating spine in $\boldsymbol{A}$ normalized to the first time point and plotted relative to time of spine elimination. Mean (red cross) and 2 SDs above and below the mean (red dashed lines) background red fluorescence intensity near the dendrite at time 0 are also plotted. C, Relative PSD-95-GFP enrichment in eliminating type Il spines in the presence of CPP (black triangles; $n=9$ ) did not change during spine elimination. PSD-95-GFP enrichment in eliminating type II spines in the presence of (PP was not significantly different from that observed for type II spines in the absence of CPP (green squares; data from Fig. 4; $p>0.6$ in all pairwise comparisons). Error bars indicate SEM.

activity-driven synapse stabilization. Proc Natl Acad Sci U S A 104:4176-4181.

El-Husseini AE, Schnell E, Chetkovich DM, Nicoll RA, Bredt DS (2000) PSD-95 involvement in maturation of excitatory synapses. Science 290:1364-1368.

Fitzjohn SM, Palmer MJ, May JE, Neeson A, Morris SA, Collingridge GL (2001) A characterisation of long-term depression induced by metabotropic glutamate receptor activation in the rat hippocampus in vitro. J Physiol 537:421-430.

Gray NW, Weimer RM, Bureau I, Svoboda K (2006) Rapid redistribution of synaptic PSD-95 in the neocortex in vivo. PLoS Biol 4:e370.

Han K, Kim MH, Seeburg D, Seo J, Verpelli C, Han S, Chung HS, Ko J, Lee HW, Kim K, Heo WD, Meyer T, Kim H, Sala C, Choi SY, Sheng M, Kim E (2009) Regulated RalBP1 binding to RalA and PSD-95 controls AMPA receptor endocytosis and LTD. PLoS Biol 7:e1000187.

Harris KM, Stevens JK (1989) Dendritic spines of CA 1 pyramidal cells in the rat hippocampus: serial electron microscopy with reference to their biophysical characteristics. J Neurosci 9:2982-2997.

Holtmaat AJ, Trachtenberg JT, Wilbrecht L, Shepherd GM, Zhang X, Knott GW, Svoboda K (2005) Transient and persistent dendritic spines in the neocortex in vivo. Neuron 45:279-291.

Hsieh H, Boehm J, Sato C, Iwatsubo T, Tomita T, Sisodia S, Malinow R (2006) AMPAR removal underlies Abeta-induced synaptic depression and dendritic spine loss. Neuron 52:831-843.

Knott GW, Holtmaat A, Wilbrecht L, Welker E, Svoboda K (2006) Spine growth precedes synapse formation in the adult neocortex in vivo. Nat Neurosci 9:1117-1124.

Kopec CD, Li B, Wei W, Boehm J, Malinow R (2006) Glutamate receptor exocytosis and spine enlargement during chemically induced

PSD-95, PSD-93, SAP-102, and SAP-97 at postsynaptic, presynaptic, and nonsynaptic sites of adult and neonatal rat visual cortex. Synapse 40:239-257.

Bastrikova N, Gardner GA, Reece JM, Jeromin A, Dudek SM (2008) Synapse elimination accompanies functional plasticity in hippocampal neurons. Proc Natl Acad Sci U S A 105:3123-3127.

Becker N, Wierenga CJ, Fonseca R, Bonhoeffer T, Nägerl UV (2008) LTD induction causes morphological changes of presynaptic boutons and reduces their contacts with spines. Neuron 60:590-597.

Bhattacharyya S, Biou V, Xu W, Schlüter O, Malenka RC (2009) A critical role for PSD-95/AKAP interactions in endocytosis of synaptic AMPA receptors. Nat Neurosci 12:172-181.

Blanpied TA, Kerr JM, Ehlers MD (2008) Structural plasticity with preserved topology in the postsynaptic protein network. Proc Natl Acad Sci U S A 105:12587-12592.

Chen X, Nelson CD, Li X, Winters CA, Azzam R, Sousa AA, Leapman RD, Gainer H, Sheng M, Reese TS (2011) PSD-95 is required to sustain the molecular organization of the postsynaptic density. J Neurosci 31:6329-6338.

Craven SE, El-Husseini AE, Bredt DS (1999) Synaptic targeting of the postsynaptic density protein PSD-95 mediated by lipid and protein motifs. Neuron 22:497-509.

De Felipe J, Marco P, Fairén A, Jones EG (1997) Inhibitory synaptogenesis in mouse somatosensory cortex. Cereb Cortex 7:619-634.

De Paola V, Holtmaat A, Knott G, Song S, Wilbrecht L, Caroni P, Svoboda K (2006) Cell type-specific structural plasticity of axonal branches and boutons in the adult neocortex. Neuron 49:861-875.

De Roo M, Klauser P, Mendez P, Poglia L, Muller D (2008) Activitydependent PSD formation and stabilization of newly formed spines in hippocampal slice cultures. Cereb Cortex 18:151-161.

Ehrlich I, Klein M, Rumpel S, Malinow R (2007) PSD-95 is required for long-term potentiation. J Neurosci 26:2000-2009.

Lawrence DG, Hopkins DA (1976) The development of motor control in the rhesus monkey: evidence concerning the role of corticomotoneuronal connections. Brain 99:235-254.

Lee S, Lee K, Hwang S, Kim SH, Song WK, Park ZY, Chang S (2006) SPIN90/WISH interacts with PSD-95 and regulates dendritic spinogenesis via an N-WASP-independent mechanism. EMBO J 25:4983-4995.

Markus EJ, Petit TL (1987) Neocortical synaptogenesis, aging, and behavior: lifespan development in the motor-sensory system of the rat. Exp Neurol 96:262-278.

Marrs GS, Green SH, Dailey ME (2001) Rapid formation and remodeling of postsynaptic densities in developing dendrites. Nat Neurosci 4:1006-1013.

Matsuzaki M, Honkura N, Ellis-Davies GC, Kasai H (2004) Structural basis of long-term potentiation in single dendritic spines. Nature 429:761-766.

Okabe S, Kim HD, Miwa A, Kuriu T, Okado H (1999) Continual remodeling of postsynaptic density and its regulation by synaptic activity. Nat Neurosci 2:804-811.

Okabe S, Miwa A, Okado H (2001) Spine formation and correlated assembly of presynaptic and postsynaptic molecules. J Neurosci 21:6105-6114.

Pak DT, Yang S, Rudolph-Correia S, Kim E, Sheng M (2001) Regulation of dendritic spine morphology by SPAR, a PSD-95-associated RapGAP. Neuron 31:289-303.

Penzes P, Johnson RC, Sattler R, Zhang X, Huganir RL, Kambampati V, Mains RE, Eipper BA (2001) The neuronal Rho-GEF Kalirin-7 interacts with PDZ domain-containing proteins and regulates dendritic morphogenesis. Neuron 29:229-242.

Pologruto TA, Sabatini BL, Svoboda K (2003) ScanImage: flexible software for operating laser scanning microscopes. Biomed Eng Online 2:13.

Prange O, Murphy TH (2001) Modular transport of postsynaptic density-95 
clusters and association with stable spine precursors during early development of cortical neurons. J Neurosci 21:9325-9333.

Rakic P, Bourgeois JP, Eckenhoff MF, Zecevic N, Goldman-Rakic PS (1986) Concurrent overproduction of synapses in diverse regions of the primate cerebral cortex. Science 232:232-235.

Roberts TF, Tschida KA, Klein ME, Mooney R (2010) Rapid spine stabilization and synaptic enhancement at the onset of behavioural learning. Nature 463:948-952.

Sekino Y, Kojima N, Shirao T (2007) Role of actin cytoskeleton in dendritic spine morphogenesis. Neurochem Int 51:92-104.

Steiner P, Higley MJ, Xu W, Czervionke BL, Malenka RC, Sabatini BL (2008) Destabilization of the postsynaptic density by PSD-95 serine 73 phosphorylation inhibits spine growth and synaptic plasticity. Neuron 60:788-802.

Stoppini L, Buchs PA, Muller D (1991) A simple method for organotypic cultures of nervous tissue. J Neurosci Methods 37:173-182.

Wise SP, Fleshman JW Jr, Jones EG (1979) Maturation of pyramidal cell form in relation to developing afferent and efferent connections of rat somatic sensory cortex. Neuroscience 4:1275-1297.
Woods G, Zito K (2008) Preparation of gene gun bullets and biolistic transfection of neurons in slice culture. J Vis Exp pii:675.

Xu T, Yu X, Perlik AJ, Tobin WF, Zweig JA, Tennant K, Jones T, Zuo Y (2009) Rapid formation and selective stabilization of synapses for enduring motor memories. Nature 462:915-919.

Yang G, Pan F, Gan WB (2009) Stably maintained dendritic spines are associated with lifelong memories. Nature 462:920-924.

Yasumatsu N, Matsuzaki M, Miyazaki T, Noguchi J, Kasai H (2008) Principles of long-term dynamics of dendritic spines. J Neurosci 28:13592-13608.

Zhou Q, Homma KJ, Poo MM (2004) Shrinkage of dendritic spines associated with long-term depression of hippocampal synapses. Neuron 44:749-757.

Zito K, Knott G, Shepherd GM, Shenolikar S, Svoboda K (2004) Induction of spine growth and synapse formation by regulation of the spine actin cytoskeleton. Neuron 44:321-334.

Zuo Y, Yang G, Kwon E, Gan WB (2005) Long-term sensory deprivation prevents dendritic spine loss in primary somatosensory cortex. Nature 436:261-265. 